\title{
Trends in Obesity and Metabolic Status Among a Health Check-Up Population in Beijing and Hunan Between 2012 and 2020
}

\section{Ying Li}

Central South University Third Xiangya Hospital

\section{Lin Yang}

Alberta Health Services

\section{Lu Yin}

Chinese Academy of Medical Sciences \& Peking Union Medical College

Qingqi Liu

Georgetown University

\section{Yaqin Wang}

Central South University Third Xiangya Hospital

Pingting Yang

Central South University Third Xiangya Hospital

Jiangang Wang

Central South University Third Xiangya Hospital

Zhiheng Chen

Central South University Third Xiangya Hospital

Xiaohui Li

Central South University

\section{Qinyu Yang}

Central South University

Yongmei He

Aerospace Central Hospital

Xin Huang ( $\nabla$ xin.huang@hunnu.edu.cn )

Hunan Normal University School of Medicine https://orcid.org/0000-0002-4874-3626

\section{Original investigation}

Keywords: obesity, metabolic status, trend, series cross-sectional study

Posted Date: September 27th, 2021

DOI: https://doi.org/10.21203/rs.3.rs-907827/v1

License: () (1) This work is licensed under a Creative Commons Attribution 4.0 International License. Read Full License 


\section{Abstract}

Background: Previous cross-sectional studies have reported the prevalence of obesity and metabolic status in China. However, the trend of change in obesity and metabolic status, especially in different sex and age groups are lacking.

Methods: In a series cross-sectional study, data on 256,782 participants surveyed between 2014 and 2020 in Beijing and 697,170 participants surveyed between 2012 and 2020 in Hunan were analyzed. Anthropometrics, blood pressure measurements, and blood tests were performed according to standard protocols. Trends in obesity and metabolic status were evaluated using the Joinpoint software to estimate annual percentage changes in slopes.

Results: Based on age- and sex-standardized values, the mean BMI values in Beijing and Hunan participants were 23.94 (95\%Cl: $23.93,23.95)$ and 23.68 (95\% Cl: $23.67,23.69) \mathrm{kg} / \mathrm{m}^{2}$, respectively. Between 2014-2020, the overall obesity prevalence among Beijing participants increased from $12.70 \%$ (95\% Cl: $12.17 \%, 13.23 \%)$ to $14.33 \%(95 \% \mathrm{Cl}: 13.97 \%, 14.70 \%)(P=0.009)$, mainly derived by the $20-39$ and $40-59$ age groups. Moreover, the prevalence of metabolically healthy obese significantly increased from $2.07 \%$ (95\% Cl: $1.84 \%, 2.30 \%)$ to $4.33 \%$ (95\% Cl: $4.13 \%, 4.53 \%)$ in Beijing. Between 2012 2020, no significant trend in obesity was found among overall Hunan participants, but the prevalence of metabolically unhealthy obese significantly increased from $5.36 \%(95 \% \mathrm{Cl}: 5.18 \%, 5.54 \%)$ to $7.35 \%$ (95\% Cl: $7.11 \%, 7.58 \%)$, mainly derived by the $20-39$ and $40-59$ age groups.

Conclusions: The trends in obesity and metabolic status were different between Hunan and Beijing. National weight control plan is needed in China, with a particular focus on young and middle-aged population.

\section{Introduction}

Obesity is a major risk factor for hypertension, diabetes, coronary heart disease, certain types of cancer, and poor mental health [1-5]. Approximately 4 million global deaths were due to high body mass index (BMI) in 2015[6]. According to the Global Burden of Disease Study, the worldwide prevalence of overweight and obesity has doubled from 1980 to 2015[7]. With the increasing spread of the global obesity pandemic, China also saw a dramatic increase in the number of overweight and obese adults [8]. For example, during 1993-2015, the prevalence of overweight, obesity and abdominal obesity increased by $14.7 \%, 11.5 \%$ and $26.7 \%$, respectively [9]. The most recent national nutrition survey indicates the prevalence of obesity was $16.4 \%$ in Chinese adults [10]. However, the annul trend of change in obesity, especially in different sex and age groups are not available.

According to the metabolic status and the BMI level, the population could be further classified into the following four phenotypes: metabolically healthy nonobese (MHNO), metabolically unhealthy nonobese (MUNO), metabolically healthy obese (MHO), and metabolically unhealthy obese (MUO) [11]. It is obvious that different phenotype present different cardiovascular and metabolic complications risk [11-13]. However, the data on trend in obesity related metabolic status in the Chinese population are lacking.

Moreover, the coronavirus disease 2019 (COVID-19) pandemic has caused significant disruption in everyday lifestyle since 2020. The changes in body weight and metabolic status that occurred during the COVID-19 pandemic in China are unknown.

In the current study, we provide new estimates of the prevalence of obesity and its metabolic status in a large number of health check-up populations from two health management centers in China.

\section{Methods}

\section{Study population}

The serial cross-sectional study population comprised more than 900,000 individuals from a mixed urban and rural area who visited health management centers in Beijing and Hunan, between 2012 and 2020. Participants with diverse socioeconomic background (public services employees, workers, self-employed persons, farmers, and others) came to health management centers to check their health status was enrolled in the current study. All participants signed informed consent forms, and the study was approved by the Ethics Committee of the Third Xiangya Hospital (2020S498).

All enrolled participants underwent a routine clinical examination. Participants recorded age, sex, current medication use, and previous medical diagnoses by themself in Beijing. Beginning in 2018, physicians reconfirmed the questionnaires during the physical examination. More detailed questionnaires, including information on exercise, smoking history, alcohol consumption, and food consumption were obtained and checked by physicians in Hunan [15]. Individuals with missing data or unreasonable values on age, height or weight were excluded. Participants with missing or unreasonable values in blood pressure『serum glucose or lipids were further excluded from metabolically status classification. The enrollment process was listed in supplementary Figure S1. Assessment methods and definitions of the clinical characteristics are detailed in the Online Appendix.

\section{Measurement and definition}

Physical examinations were conducted with the same methods described in our previous study [16]. Briefly, blood pressure, height and weight were measured by trained physicians. Participants were categorized into nonobese $\left(<28 \mathrm{~kg} / \mathrm{m}^{2}\right)$ and obese $\left(\geq 28 \mathrm{~kg} / \mathrm{m}^{2}\right) \mathrm{groups}$ [17]. The definitions of the 
metabolic unhealthy status are detailed in the Online Appendix. As mentioned above [11], the participants were further classified into the MHNO MUNO, MHO, and MUO. phenotypes.

\section{Laboratory measurements}

Fasting venous blood samples were collected and were immediately processed and analyzed at the clinical laboratory of Third Xiangya Hospital or Aerospace Center Hospital, as detailed in the Online Appendix.

\section{Statistical analysis}

Continuous variables were expressed as means with 95\% confidence intervals (95\% Cls), and categorical variables were expressed as percentages with $95 \% \mathrm{Cls}$, and differences among different BMI groups were tested by analysis of variance and the Chi-square test, respectively. First, restricted cubic spline regression models were used to test the overall and nonlinear association between survey years and BMI, HDL-C, TG, SBP, DBP and FSG. Then, if the overall association were significant with no significant nonlinear association, linear trends were further evaluated in regression models. Means of BMI and metabolic status were further stratified by location and sex because of significant 3-way interactions. Trends in prevalence of obesity and its metabolic status were evaluated using the Joinpoint software, and annual percentage changes (APCs) in slopes were reported. Moreover, the prevalence of obesity and its metabolic status were stratified by location, age group (20-39, 40-59, 60 and older years) and sex because of significant 4-way interactions. The prevalence of obesity, MHNO, MUNO, MHO and MUO and the mean levels of BMI, total cholesterol (TC), HDL-C, and TG, SBP, DBP, and FSG were all estimated for females and males after age standardization according to the population distribution in China in 2010. The weights were $0.4385,0.3860$, and 0.1755 for the $20-39,40-59$, and over 60 years old groups, respectively. When calculating estimates for each survey years, sex was additionally adjusted, and weights for females and males were the same as 0.5 . A two-sided $P<0.05$ was considered to be statistically significant. SAS version 9.4 (SAS Institute Inc) was used for analyses.

\section{Results}

\section{Characteristics of selected study participants}

A total of 256,782 participants were surveyed from 2014 to 2020 in Beijing and 697,170 participants surveyed from 2012 to 2020 in Hunan were enrolled in our study.

Overall, the mean BMI was 23.91 (95\% Cl: $23.90,23.92) \mathrm{kg} / \mathrm{m}^{2}$ and 108,914 participants $(11.41 \%$ [95\% Cl: $\left.11.34 \%, 11.47 \%]\right)$ were obese. Table 1 shows the demographic and clinical characteristics of the study participants by obesity status. Compared to the non-obese, participants in the obese group were more likely to be male, from Beijing, older, with higher level of TC, TC, LDL-C, and FSG, having elevated blood pressure, with hypertension, diabetes or hyperlipidemia $(p<0.001$, Table 1$)$.

\section{Trends in BMI and obesity}

The age- and sex-standardized mean BMI levels between Beijing and Hunan participants were 23.94 (95\% Cl: 23.93, 23.95) and 23.68 (95\% Cl: 23.67, $23.69) \mathrm{kg} / \mathrm{m}^{2}$, respectively (Table 2). During 2014- 2020, the BMI levels in Beijing showed significant nonlinear changes by surveyed year ( $\left.P<0.001\right)$ and grew rapidly after 2018 (Figure 1). A nonlinear trend in BMI level among female from Hunan was also observed, which increased from 2012 to 2016 and declined after 2016. However, a significant and linear increasing trend in BMI trend was observed among the Hunan male population $(P<0.001)$ (Figure 1). Due to the interaction between the BMI and age, we further stratified the population by age. As a result, the BMI level was found to significantly increased among 20-39 aged groups in both area, and the trends between Hunan male and Beijing female groups were linear. Among the 40-59 years old, nonlinear upward trends were observed in both sexes from Beijing and Hunan. On the other hand, a significant and downward trend in BMI was found among female participants over 60 from Hunan (Table S1 and Figure S2).

Between 2014 and 2020, the prevalence of obesity among Beijing participants increased from 12.70\% (95\% Cl: 12.17\%, 13.23\%) to 14.33\% (95\% Cl: $13.97 \%, 14.70 \%)(P=0.009)$ (Fig 2A). The obesity prevalence among Beijing females and males showed similar annual percentage changes. Among the 20-39 and 40-59 age group, the annually percentage changes were 6.07 (95\% Cl: 3.97, 8.21) and 3.17 (95\% Cl: 1.02, 5.37), respectively. However, the trends of obesity in the over 60 years age group were nonsignificant $(P=0.668)$ (Fig 2B-D).

The trends of obesity among Hunan participants were different from those among Beijing participants. Between 2012 and 2020 , no significant trend in obesity was found among the total sample of Hunan participants $(P=0.240)$ (Fig 2A). After stratification by age and sex, the prevalence of obesity among females and males aged $20-39$ years was found to increase from $1.87 \%(95 \% \mathrm{Cl}, 1.63 \%, 2.11 \%)$ to $2.99 \%(95 \% \mathrm{Cl}, 2.69 \%, 3.29 \%)$ and from $13.10 \%(95 \% \mathrm{Cl}, 12.53 \%, 13.67 \%)$ to $16.04 \%(95 \% \mathrm{Cl}, 15.34 \%, 16.74 \%)$, respectively (Fig $2 \mathrm{~B})$. However, obesity among over 60 years old Hunan females showed significant decreasing trends $(P<0.006)$, and among 40-59 years aged Hunan females, the prevalence of obesity increased between 2012 and 2014 (slope=0.12 (95\% Cl: -0.07,0.30), $P=0.17$ ), and decreased after 2014 (slope=-0.03 (95\% Cl: $-0.06,0.00), P=0.04)($ Fig 2C, D).

\section{Trends in metabolic factors}


Overall, a total of 908,311 persons were included for metabolic analysis. The age-adjusted means of FSG, TG, HDL-c, SBP and DBP are listed in Supplementary Tables S2-S6. Overall, the change trend in metabolic factors was similar between different sexes but show regional differences expect for in FSG, which were both rising in the two places (Figure S3). The TG levels showed a downward trend in 2014-2018 and an upward trend in 20182020 among Beijing population, and in the Hunan region it showed a non-linear monotonous rising trend (Figure S4). The HDL-c levels had opposite trends in the two places, with Beijing rising and Hunan declining (Figure S5). The SBP levels showed an upward trend in Beijing, and in the Hunan region it showed a downward trend in 2012- 2016 and an upward trend in 2016-2020 (Figure S6). The DBP levels rose first and then fell in Beijing, and fell first and then rose in Hunan (Figure S7).

\section{Trends of obesity phenotypes}

Between 2014 and 2020, among Beijing participants, the prevalence of the MUNO subtype significantly decreased from 36.03\% (95\% Cl: 35.16\%, $36.90 \%)$ to $27.85 \%(95 \% \mathrm{Cl}: 27.31 \%, 28.39 \%)$ with an annual percentage change of $-6.53(95 \% \mathrm{Cl}:-12.33$, -0.34$)$ (Fig 3B), but the trends in MUO were nonsignificant ( $\mathrm{p}=0.384$ ) (Fig 3D). However, the trends in MHNO were nonsignificant and the prevalence of MHO increased significantly from $2.07 \%$ (95\% Cl: $1.84 \%, 2.30 \%)$ to $4.33 \%$ (95\% Cl: $4.13 \%, 4.53 \%)$ (Fig 3C). All obesity phenotypes in different sex and age groups among Beijing participants showed similar annual percentage changes (Fig S8).

Unlike the trends in obesity phenotypes among Beijing participants, between 2012 and 2020, the prevalence of the metabolically unhealthy subtypes MUNO and MUO among Hunan participants increased significantly from $21.92 \%$ (95\% Cl: $21.55 \%, 22.30 \%)$ to $29.58 \%$ (95\% Cl: $29.10 \%, 30.07 \%$ ) and from $5.36 \%(95 \% \mathrm{Cl}: 5.18 \%, 5.54 \%)$ to $7.35 \%$ (95\% Cl: 7.11\%, 7.58\%), respectively (Fig 3B, D). Meanwhile, the prevalence of the metabolically healthy subtypes MHNO and MHO decreased significantly from 69.24\% (95\% Cl: 68.57\%, 69.92\%) to 60.21\% (95\% Cl: 59.55\%, 60.88\%) and from 3.47\% (95\% Cl: $3.32 \%, 3.61 \%$ ) to $2.86 \%$ (95\% Cl: $2.71 \%, 3.00 \%$ ), respectively (Fig 3A, C). Among Hunan participants aged $40-59$ and over 60 years old, females and males showed a similar annual percentage change in all obesity phenotypes (Fig S8), but among the 20-39 year age group, the prevalence of MUNO and MUO in females showed a greater annual percentage increase than that in males $(14.84$ [95\% Cl: 9.40, 20.56] vs 5.18 [95\% Cl: $2.62,7.81]$ and 15.02 [95\% Cl: $13.55,16.51]$ vs 5.32 [95\% Cl: 3.74, 6.92]) (Fig S8).

\section{Discussion}

The present analyses show that the mean BMI and the prevalence of obesity in Beijing were significantly higher than that in Hunan. However, compared to Beijing, marked changes in metabolic abnormalities occurred in Hunan from 2012 to 2020 . The mean BMI, and the prevalence of obesity increased significantly in Beijing, driven primarily by 20-39 and 40-59 years adult males and females from 2014 to 2020 . In Hunan, the mean BMI increased significantly, but the overall obesity prevalence was stable. Moreover, the proportion of MUO increased, especially in 20-39 years females and $20-39$ and $40-59$ years male population.

In previous study, the age-standardized mean BMI increased by $2.0 \mathrm{~kg} / \mathrm{m}^{2}$ from 1993 to 2015 in China [9]. Our study further confirms the mean BMI continued to increase in both Beijing and Hunan area from 2012 to 2020. But the trend in BMI differed between Beijing and Hunan area. The mean BMI level in Beijing increased rapidly after 2018, whereas in Hunan, it increased obviously from 2012 to 2016. Moreover, unlike the other study [9], the increased mean BMI level was mainly derived by young and middle aged population, especially in Beijing.

The trend in obesity prevalence in our study was inconsistent with other studies. Globally, the prevalence of obesity increased from $5 \%$ in 1980 to $10.1 \%$ in males and from $8.9-14.8 \%$ in females in 2015 [18]. Based on cross-sectional surveys, the prevalence of obesity increased from $4.2-15.7 \%$ in the Chinese population between 1993 and 2015 [9]. In the other regional studies, the prevalence of obesity in Jilin City and Nanjing City were increased significantly by $3.6 \%$ and $4.0 \%$ for male, and by $5.6 \%, 1.5 \%$ for female, from 2007 to 2013 and 2008 to 2016 in China, respectively [19]. However, the increase in obesity in Beijing was 3.47\% per year, while no significant increasing trend was found in Hunan Province in current study. In Wang's study, the change in the prevalence of obesity in migrants was - 0.6\% in Sichuan Province from 2007-2015 [20]. The geographical position of Hunan Province borders Sichuan Province; thus, the demographic characteristics might be similar between those two areas.

In addition, aged 20-39, and 40-59 populations drove the increasing trend of the prevalence of obesity in Beijing. The turning point of the prevalence of obesity in females aged 40-59 and the decline trend in females over 60 years old have evened out the rising rate of in both females and males aged 20-39, thus the overall obesity rate did not shown an increasing trend in Hunan. In previous studies, the increasing trend in obesity was driven by all age subgroups, especially by $40-80$ aged population, surveyed before 2015 [21]. Our study indicated that more attention should be given to the young and middle-aged populations in China.

It is demonstrated that Covid-19 has made the obesity epidemic worse in the world [22]. For example, a study conducted in Italy has shown home isolation and adverse mental health burden linked to the COVID-19 pandemic were associated with significant weight gain in 2020 [23]. In the current study, neither the mean BMI nor the prevalence of obesity rate showed a significant change in 2020 that deviate from the previous trend. In our view, the rapidly controlled COVID-19 pandemic might not have had a large impact on weight in China. Some previous reports of weight gain during the pandemic might have obtained significant results because they did not compare the results to previous trends.

In our study, the prevalence of $\mathrm{MHO}$ was $4.33 \%$ and $2.95 \%$ in Beijing and Hunan in 2020, respectively. In the China Kadoorie Biobank study, the MHO phenotype accounted for $3.3 \%$ of the total population during the period 2004 to 2008 in China [24]. According to a previous study, the prevalence of

Page $4 / 12$ 
$\mathrm{MHO}$ has been shown to range between $4.2 \%$ and $13.6 \%$ in a random sample from a Chinese adult population, depending on the definition used for $\mathrm{MHO}$ [25]. The trends in the prevalence of obesity metabolic status differed between Beijing and Hunan. Overall, the increasing trend in the prevalence of obesity was predominantly driven by MHO in all age groups in Beijing. During the period from 1973-1980, Keyes and Reuben Andres suggested that $\mathrm{MHO}$ could be benign and not contribute to cardiovascular risk [26]. However, an increasing number of studies have demonstrated MHO is indeed associated with an increased risk of cardiovascular disease, chronic kidney disease, nonalcoholic fatty liver disease and so on [27-29]. Therefore, $\mathrm{MHO}$ could be a risk factor for chronic disease [30] and finally transit to MUO [31], if timely intervention is not performed in Beijing area. In support, although the overall prevalence of obesity was stable, the prevalence of MUO phenotype has increased, while the prevalence of MHO has decreased, especially in young and middle-aged groups in Hunan. A previous study done in Shanghai adults indicated the prevalence of metabolism problem was doubled with an increase in metabolically unhealthy overweight from 2002 to 2017 as well [32]. There is no doubt that MUO has the greatest impact on health. Therefore, residents in Hunan should pay more attention to metabolic status.

High energy intake, especially sugar drinks and other energy-dense foods, and low levels of physical activity contributed to the increasing trend of obesity in China [33-34]. In the current study, the relatively slow growth trend in Beijing and the stable trend in Hunan may be due to the following reasons: First, recognizing the immediacy of chronic disease challenges, the 'China Healthy Lifestyle for All' initiative launched in 2007 was developed to raise awareness of a range of preventive health issues, such as knowledge of dietary guidelines, and the adoption of health promoting behaviors. Currently, much evidence suggests a positive role on healthy lifestyle action after 2015[35]. Thus, the good knowledge of healthy lifestyles may help control weight in Chinese adults. Second, the participants of the current study were from a health check-up population who received health education and guidance on weight intervention from the Health Management Center. Therefore, the result may not be representative of the data of the national epidemiologic survey.

Our study has several strengths. First, it included a large sample size (>900,000 participants) from Beijing and Hunan (North and Central South regions of China). Second, the surveys were performed every year between 2012 and 2020 to facilitate the analyses for annul trend of change. Third, we also used physical examination data to analyze the metabolic status and further understand the types of obesity. However, several limitations

should be considered. First, as mentioned above, health check-up populations may not represent the majority of the Chinese population. Second, waist circumference was not a regular measurement in Beijing thus was not analyzed in the current study. Third, the questionnaires was obtain from participants under voluntary principle before 2018 in Beijing, thus some information such as medication use history may lower than the real world data. Fourth, lifestyle was associated with obesity and metabolism disorder [36]. But we were unable to examine the roles of nutrition and lifestyle factors (e.g., physical activity and sleep duration) on obesity trends because these data were not continuously collected in Beijing.

\section{Conclusions}

The trends in the prevalence of obesity and metabolic status were different between Beijing and Hunan population. The Beijing area was dominated by the growth of young and middle-aged obesity and $\mathrm{MHO}$ phenotype, while although the overall obesity rate was stable in Hunan, the proportion of MUO increased, especially in young and middle-aged population. The weight control plan should be pay more attention to young and middle-aged population in China.

\section{Declarations}

\section{Acknowledgments}

The authors gratefully acknowledge the voluntary participation of all study subjects.

\section{Funding}

This work was supported by funding from the National Natural Science Foundation of China (81973324 to YL, 81872685 to XH, and 82003537 to XH), Hunan Young Talent grant (2020RC3063 to YL), Hunan Science Foundation (2020JJ5858 to YL, 2020JJ4439 to XH) and the Wisdom Accumulation and Talent Cultivation Project of the Third XiangYa hosipital of Central South University (YX202002 to YL). The funders had no role in study design, data collection and analysis, decision to publish, or preparation of the manuscript.

\section{Authors' contributions}

YL, Lin Yang, Lu Yin, QL and XH produced data for analysis. YL, Lin Yang, XH and YH wrote the manuscript. IG, AE and JN designed the study. PY, YW, JW, ZC, XL, QY and YH included patients for the study. All authors reviewed and edited the manuscript. AE, IG, and JN handled funding and supervision. All authors read and approved the final manuscript.

\section{Availability of data and materials}

The datasets generated and/or analyzed during the current study are not publicly available due the sensitive nature of the data.

\section{Ethics approval and consent to participate}


All participants signed informed consent forms, and the study was approved by the Ethics Committee of the Third Xiangya Hospital (2020-S498).

\section{Consent for publication}

Not applicable.

\section{Competing interests}

There is no conflict of interest relevant to this manuscript.

\section{References}

1. Must A, Spadano J, Coakley EH, Field AE, Colditz G, Dietz WH. The disease burden associated with overweight and obesity. JAMA. 1999;282:1523-9.

2. Lauby-Secretan B, Scoccianti C, Loomis D, Grosse Y, Bianchini F, Straif K, et al. Body Fatness and Cancer-Viewpoint of the IARC Working Group. N Engl J Med. 2016;375:794-8.

3. Ayton A, Ibrahim A. Obesity is a public health emergency. BMJ. 2019;366:I5463.

4. Anandacoomarasamy A, Caterson I, Sambrook P, Fransen M, March L. The impact of obesity on the musculoskeletal system. Int J Obes (Lond). 2008;32:211-22.

5. Anstey KJ, Cherbuin N, Budge M, Young J. Body mass index in midlife and late-life as a risk factor for dementia: a meta-analysis of prospective studies. Obes Rev. 2011;12:e426-437.

6. GBD 2015 Obesity Collaborators, Afshin A, Forouzanfar MH, Reitsma MB, Sur P, Estep K, et al. Health Effects of Overweight and Obesity in 195 Countries over 25 Years. N Engl J Med. 2017;377:13-27.

7. Global Burden of Disease Study 2015. Global burden of disease study 2015 (GBD 2015) obesity and overweight prevalence 1980-2015. Seattle, United States: Institute for Health Metrics and Evaluation (IHME); 2017.

8. Pan X-F, Wang L, Pan A. Epidemiology and determinants of obesity in China. Lancet Diabetes Endocrinol. 2021;9:373-92.

9. Ma S, Xi B, Yang L, Sun J, Zhao M, Bovet P. Trends in the prevalence of overweight, obesity, and abdominal obesity among Chinese adults between 1993 and 2015. International Journal of Obesity. 2021;45:427-37.

10. The State Council Information Office of the People's Republic of China. Press briefing for the Report on Chinese Residents' Chronic Diseases and Nutrition 2020. December 24, 2020.

11. Hinnouho G-M, Czernichow S, Dugravot A, Nabi H, Brunner EJ, Kivimaki M, et al. Metabolically healthy obesity and the risk of cardiovascular disease and type 2 diabetes: the Whitehall II cohort study. Eur Heart J. 2015;36:551-9.

12. Eckel N, Mühlenbruch K, Meidtner K, Boeing H, Stefan N, Schulze MB. Characterization of metabolically unhealthy normal-weight individuals: Risk factors and their associations with type 2 diabetes. Metabolism. 2015;64:862-71.

13. Hinnouho G-M, Czernichow S, Dugravot A, Batty GD, Kivimaki M, Singh-Manoux A. Metabolically healthy obesity and risk of mortality: does the definition of metabolic health matter? Diabetes Care. 2013;36:2294-300.

14. Ko S-H, Baeg MK, Ko SY, Jung HS, Kim P, Choi M-G. Obesity and Metabolic Unhealthiness Have Different Effects on Colorectal Neoplasms. J Clin Endocrinol Metab. 2017;102:2762-9.

15. Yang P, Chen Z, Yin L, Peng Y, Li X, Cao X, et al. Salt intake assessed by spot urine on physical examination in Hunan, China. Asia Pac J Clin Nutr. 2019;28:845-56.

16. Lu Y, Pechlaner R, Cai J, Yuan H, Huang Z, Yang G, et al. Trajectories of Age-Related Arterial Stiffness in Chinese Men and Women. J Am Coll Cardiol. 2020;75:870-80.

17. National Health and Family Planning Commission of the People's Republic of China. WST 428-2013 Criteria of weight for adults. Standards Press of China: Beijing.

18. NCD Risk Factor Collaboration (NCD-RisC). Trends in adult body-mass index in 200 countries from 1975 to 2014 : a pooled analysis of 1698 population-based measurement studies with 19.2 million participants. Lancet. 2016;387:1377-96.

19. Sun J, Zhou W, Gu T, Zhu D, Bi Y. A retrospective study on association between obesity and cardiovascular risk diseases with aging in Chinese adults. Sci Rep. 2018;8:5806.

20. Zhang J, Wan S, Zhang B, Dong F, Pan L, Yihuo W, et al. Twenty-year time trends in hypertension prevalence in Yi people of China: three successive cross-sectional studies, 1996-2015. BMJ Open. 2018;8:e022714.

21. Mi Y-J, Zhang B, Wang H-J, Yan J, Han W, Zhao J, et al. Prevalence and Secular Trends in Obesity Among Chinese Adults, 1991-2011. Am J Prev Med. 2015;49:661-9.

22. Senthilingam M. Covid-19 has made the obesity epidemic worse, but failed to ignite enough action. BMJ. 2021;372:n411.

23. Pellegrini M, Ponzo V, Rosato R, Scumaci E, Goitre I, Benso A, et al. Changes in Weight and Nutritional Habits in Adults with Obesity during the "Lockdown" Period Caused by the COVID-19 Virus Emergency. Nutrients. 2020;12:E2016. 
24. Gao M, Lv J, Yu C, Guo Y, Bian Z, Yang R, et al. Metabolically healthy obesity, transition to unhealthy metabolic status, and vascular disease in Chinese adults: A cohort study. PLoS Med. 2020;17:e1003351.

25. Liu C, Wang C, Guan S, Liu H, Wu X, Zhang Z, et al. The Prevalence of Metabolically Healthy and Unhealthy Obesity according to Different Criteria. Obes Facts. 2019;12:78-90.

26. Sims EA. Are there persons who are obese, but metabolically healthy? Metabolism. 2001;50:1499-504.

27. Opio J, Croker E, Odongo GS, Attia J, Wynne K, McEvoy M. Metabolically healthy overweight/obesity are associated with increased risk of cardiovascular disease in adults, even in the absence of metabolic risk factors: A systematic review and meta-analysis of prospective cohort studies. Obes Rev. 2020;21:e13127.

28. Chang Y, Jung H-S, Cho J, Zhang Y, Yun KE, Lazo M, et al. Metabolically Healthy Obesity and the Development of Nonalcoholic Fatty Liver Disease. Am J Gastroenterol. 2016;111:1133-40.

29. Stanford FC, Butsch WS. Metabolically Healthy Obesity and Development of Chronic Kidney Disease. Ann Intern Med. 2016;165:742-3.

30. Kim HS, Lee J, Cho YK, Park J-Y, Lee WJ, Kim Y-J, et al. Differential Effect of Metabolic Health and Obesity on Incident Heart Failure: A Nationwide Population-Based Cohort Study. Front Endocrinol (Lausanne). 2021;12:625083.

31. Gao M, Lv J, Yu C, Guo Y, Bian Z, Yang R, et al. Metabolically healthy obesity, transition to unhealthy metabolic status, and vascular disease in Chinese adults: A cohort study. PLoS Med. 2020;17:e1003351.

32. Yang Y, Li Y, Pei J, Cheng M, Xu W, Shi Y. Dynamic changes in metabolic health status in Chinese adults: Multiple population-based surveys in Shanghai, China. J Diabetes Investig. 2021;

33. Popkin BM. Synthesis and implications: China's nutrition transition in the context of changes across other low-and middle-income countries. Obes Rev. 2014;15 Suppl 1:60-7.

34. Li Y, Wang DD, Ley SH, Howard AG, He Y, Lu Y, et al. Potential Impact of Time Trend of Life-Style Factors on Cardiovascular Disease Burden in China. J Am Coll Cardiol. 2016;68:818-33.

35. Zhang J, Astell-Burt T, Seo D-C, Feng X, Kong L, Zhao W, et al. Multilevel evaluation of "China Healthy Lifestyles for All", a nationwide initiative to promote lower intakes of salt and edible oil. Prev Med. 2014;67:210-5.

36. Sotos-Prieto M, Ortolá R, Ruiz-Canela M, Garcia-Esquinas E, Martínez-Gómez D, Lopez-Garcia E, et al. Association between the Mediterranean lifestyle, metabolic syndrome and mortality: a whole-country cohort in Spain. Cardiovasc Diabetol. 2021;20:5.

\section{Tables}

Table 1 Characteristics of selected study participants by obesity status 


\begin{tabular}{|c|c|c|c|}
\hline \multicolumn{2}{|l|}{ Characteristics } & $\begin{array}{l}\text { Non-obesity } \\
\mathrm{N}(\%) / \text { mean } \pm \text { SD }\end{array}$ & $\begin{array}{l}\text { Obesity } \\
\mathrm{N}(\%) / \text { mean } \pm \text { SL }\end{array}$ \\
\hline \multirow[t]{2}{*}{ Location $^{\star *}$} & Beijing & $220829(26.13)$ & $35953(33.01)$ \\
\hline & Hunan & $624209(73.87)$ & 72961(66.99) \\
\hline \multirow[t]{2}{*}{$\operatorname{Sex}^{* *}$} & Female & $386537(45.74)$ & $23584(21.65)$ \\
\hline & Male & $458501(54.26)$ & $85330(54.26)$ \\
\hline \multicolumn{2}{|c|}{$\mathrm{Age}^{\star *}$, year $(\mathrm{N}=953,952)$} & $44.56 \pm 14.79$ & $46.13 \pm 14.20$ \\
\hline \multicolumn{2}{|c|}{$\mathrm{BMI}, \mathrm{kg} / \mathrm{m}^{2}(\mathrm{~N}=953,952)$} & $23.11 \pm 2.65$ & $30.10 \pm 2.10$ \\
\hline \multicolumn{2}{|c|}{$\mathrm{TG}^{\star \star}, \mathrm{mmol} / \mathrm{L}(\mathrm{N}=933,854)$} & $1.53 \pm 1.39$ & $2.46 \pm 2.15$ \\
\hline \multicolumn{2}{|c|}{$\mathrm{TC}^{\star \star}, \mathrm{mmol} / \mathrm{L}(\mathrm{N}=933,872)$} & $4.90 \pm 0.95$ & $5.14 \pm 1.01$ \\
\hline \multicolumn{2}{|c|}{$\mathrm{HDL}-\mathrm{c}^{\star *}, \mathrm{mmol} / \mathrm{L}(\mathrm{N}=923,832)$} & $1.39 \pm 0.39$ & $1.15 \pm 0.29$ \\
\hline \multicolumn{2}{|c|}{ LDL-c ${ }^{\star \star}, \mathrm{mmol} / \mathrm{L}(\mathrm{N}=923,715)$} & $2.72 \pm 0.80$ & $2.84 \pm 0.85$ \\
\hline \multicolumn{2}{|c|}{$\mathrm{SBP}^{\star \star}, \mathrm{mmHg}(\mathrm{N}=938,969)$} & $121.36 \pm 16.31$ & $132.81 \pm 16.08$ \\
\hline \multicolumn{2}{|c|}{$\mathrm{DBP}^{\star \star}, \mathrm{mmHg}(\mathrm{N}=938,985)$} & $74.79 \pm 10.92$ & $83.27 \pm 11.57$ \\
\hline \multicolumn{2}{|c|}{$\mathrm{FSG}^{\star \star}, \mathrm{mmol} / \mathrm{L}(\mathrm{N}=942,864)$} & $5.35 \pm 1.18$ & $5.84 \pm 1.61$ \\
\hline \multirow[t]{2}{*}{ Hypertension ${ }^{\star \star}$} & No & $681,807(81.93)$ & $62,506(58.53)$ \\
\hline & Yes & $150,342(18.07)$ & $44,278(41.47)$ \\
\hline \multirow[t]{2}{*}{ Diabetes ${ }^{\star \star}$} & No & $79,1011(94.75)$ & $94,512(87.50)$ \\
\hline & Yes & $43,841(5.25)$ & $13,500(12.50)$ \\
\hline \multirow[t]{2}{*}{ Dyslipidemia ** } & No & $546,177(66.82)$ & $40,345(38.01)$ \\
\hline & Yes & $271,260(33.18)$ & $65,785(61.99)$ \\
\hline
\end{tabular}

** $p<0.01$

Abbreviation: SD, standard deviation; BMI, body mass index; SBP, systolic blood pressure; DBP, diastolic blood pressure; FSG, fasting serum glucose; TG, triglyceride; TC, total cholesterol; LDL-c, low-density lipoprotein cholesterol; HDL-c, high-density lipoprotein cholesterol

Table 2 Age-adjusted mean and 95\% confidence interval of BMI levels among Chinese adults over 20 years old in Beijing and Hunan Province, 20122020 * 


\begin{tabular}{|c|c|c|c|c|c|c|}
\hline \multirow[t]{2}{*}{ Year } & \multicolumn{2}{|l|}{ Beijing } & & $\mathrm{BMI}, \mathrm{kg} / \mathrm{m}^{2}($ mean $[95 \% \mathrm{Cl}])$ & Hunan & \\
\hline & All $\$$ & Female & Male & All $\$$ & Female & Male \\
\hline 2012 & & & & $23.46(23.44,23.49)$ & $22.33(22.30,22.37)$ & $24.59(24.56,24.62)$ \\
\hline 2013 & & & & $23.63(23.61,23.66)$ & $22.46(22.43,22.49)$ & $24.80(24.78,24.83)$ \\
\hline 2014 & $23.85(23.80,23.90)$ & $22.76(22.69,22.84)$ & $24.94(24.87,25.00)$ & $23.74(23.72,23.76)$ & $22.60(22.57,22.63)$ & $24.88(24.85,24.91)$ \\
\hline 2015 & $23.67(23.63,23.71)$ & $22.59(22.54,22.65)$ & $24.75(24.70,24.80)$ & $23.75(23.73,23.77)$ & $22.59(22.56,22.62)$ & $24.90(24.87,24.93)$ \\
\hline 2016 & 23.78(23.74,23.82) & $22.71(22.66,22.76)$ & $24.85(24.80,24.90)$ & $23.61(23.58,23.63)$ & $22.46(22.43,22.49)$ & $24.75(24.72,24.78)$ \\
\hline 2017 & 23.78(23.75,23.82) & $22.68(22.63,22.73)$ & $24.89(24.85,24.93)$ & $23.68(23.66,23.70)$ & $22.54(22.51,22.57)$ & $24.82(24.79,24.85)$ \\
\hline 2018 & $23.97(23.94,24.00)$ & $22.92(22.87,22.96)$ & $25.02(24.98,25.06)$ & $23.67(23.65,23.70)$ & $22.46(22.43,22.49)$ & $24.89(24.86,24.92)$ \\
\hline 2019 & $24.04(24.02,24.07)$ & $22.99(22.95,23.03)$ & $25.10(25.06,25.13)$ & $23.79(23.77,23.81)$ & $22.60(22.57,22.63)$ & $24.98(24.95,25.01)$ \\
\hline 2020 & $24.16(24.13,24.19)$ & $22.98(22.94,23.03)$ & $25.34(25.29,25.38)$ & $23.73(23.70,23.75)$ & $22.49(22.45,22.53)$ & $24.96(24.93,25.00)$ \\
\hline $\begin{array}{l}p \text { for overall } \\
\text { trend }\end{array}$ & $<.0001$ & $<.0001$ & $<.0001$ & $<.0001$ & $<.0001$ & $<.0001^{\#}$ \\
\hline $\begin{array}{l}p \text { for non- } \\
\text { linear trend }\end{array}$ & $<.0001$ & $<.0001$ & $<.0001$ & $<.0001$ & $<.0001$ & 0.207 \\
\hline Subtotal & $23.94(23.93,23.95)$ & $22.86(22.84,22.88)$ & $25.02(25.01,25.04)$ & $23.68(23.67,23.69)$ & $22.51(22.50,22.53)$ & $24.84(24.83,24.85)$ \\
\hline
\end{tabular}

* Estimates are age-adjusted to the 2010 Chinese Census population using age groups 20-39, 40-59, and 60 or older; $\$$ additional adjusted by sex; \# $P$ for linear trends $<0.001$

\section{Figures}
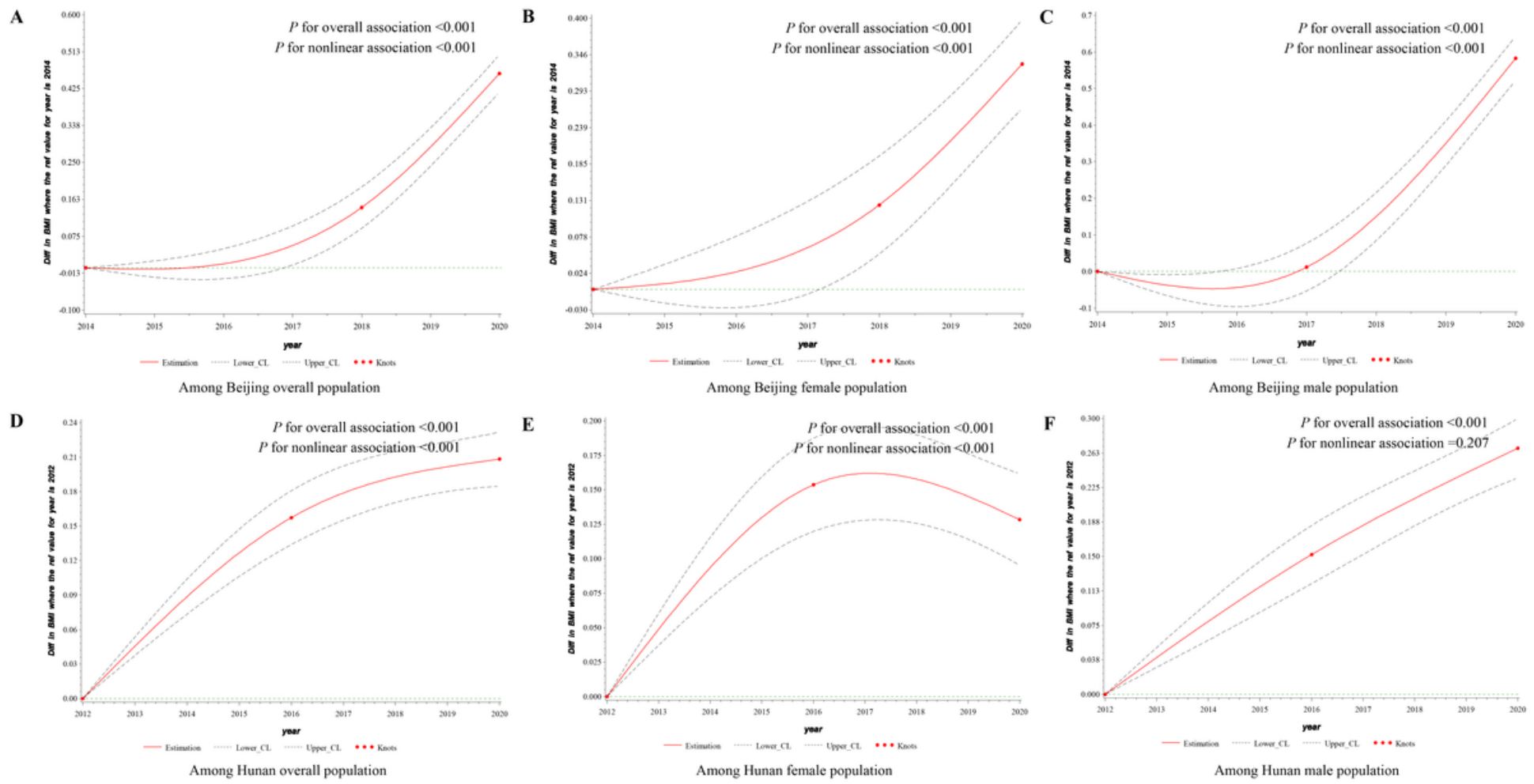

Figure 1

Association between surveyed years and BMI among Beijing and Hunan adults over 20 years old during 2012-2020 Surveyed year was coded using RCS function with three knots located at the 5th, 50th, and 95th percentiles of the distribution of survey years. Y-axis represents the difference (Diff) in BMI. Referenced year in (A) (B) (C) was 2014 and in (D) (E) (F) was 2012. Dashed lines are 95 per cent confidence intervals. Knots are represented by 
dots. In (A) and (D), age and sex were included as adjustment variables.; In (B), (C), (E) and (F), age was included as adjustment variable. Abbreviation: BMI, body mess index

A

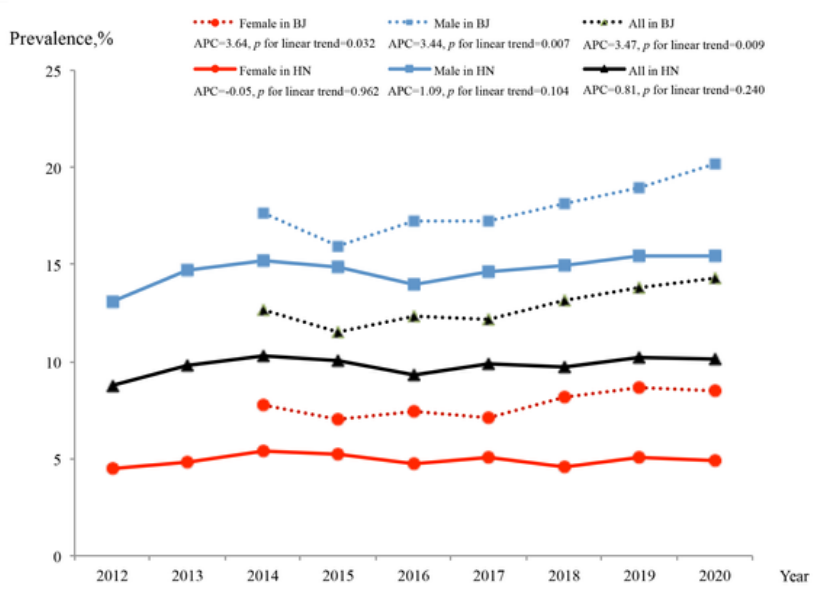

C

Age standarized obesity prevalence among Beijing and Hunan adults over 20 years old, 2012-2020

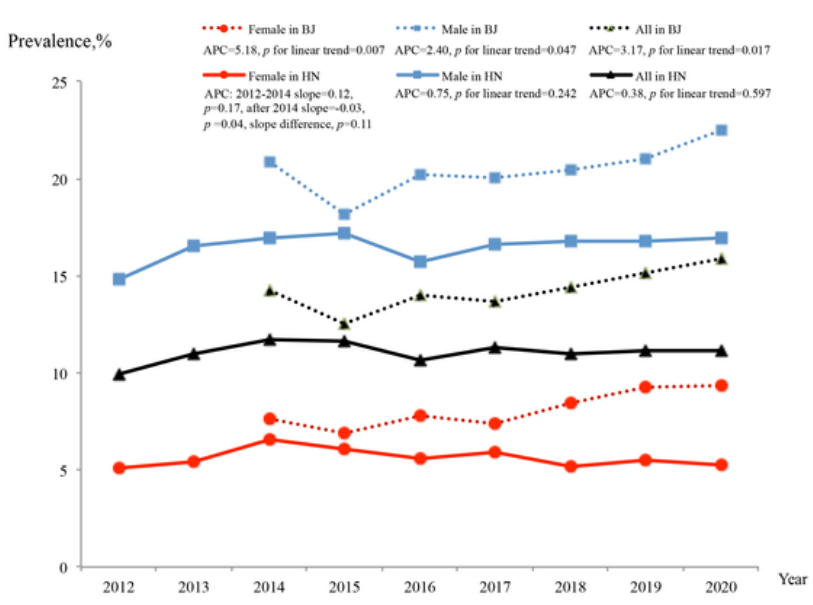

Obesity prevalence among Beijing and Hunan adults aged 40-59 years old, 2012-2020
B

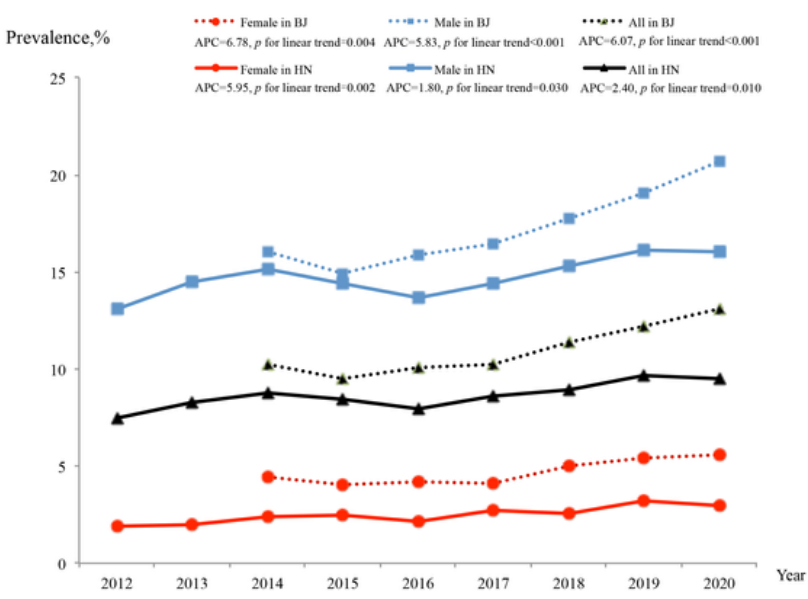

Obesity prevalence among Beijing and Hunan adults aged 20-39 years old, 2012-2020

D

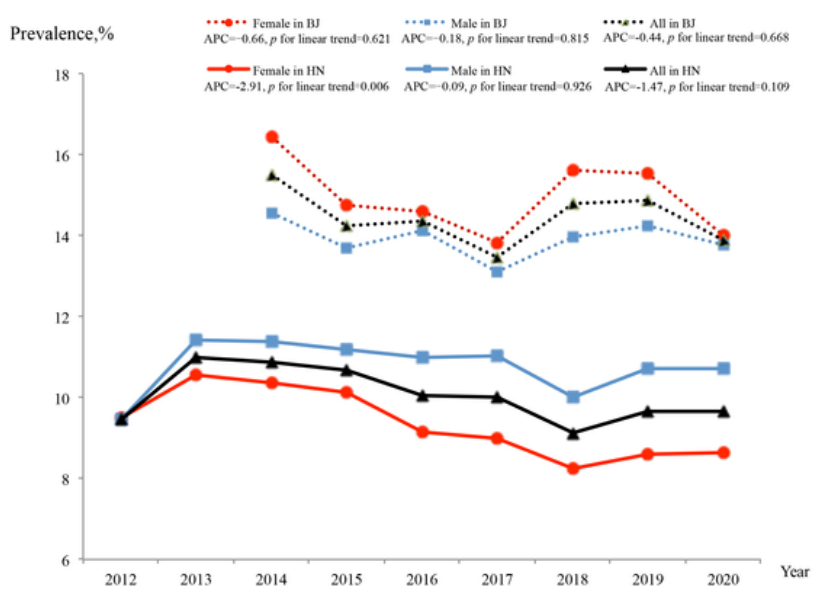

Obesity prevalence among Beijing and Hunan adults over 60 years old, 2012-2020

\section{Figure 2}

Trend in prevalence of obesity in Beijing and Hunan Adults from 2012 to 2020 Estimates by different sex are age-adjusted to the 2010 Chinese Census population using age groups $20-39,40-59$, and 60 or older and estimates for overall population are additional adjusted by sex; Significant linear trends $(\mathrm{P}<0.05)$ for the following groups :(1) increased age-standardized obesity among all sex groups from Beijing; (2) increased obesity among all 20-39 aged groups from Beijing and Hunan; (3) increased obesity among all 40-59 aged groups from Beijing ( $P<0.05)$; (4) among 40-59 aged female from Hunan, obesity during 2012-2014 showed increased level (slope=0.12 (95\% Cl: -0.07,0.30), P =0.17), and level decreased after 2014 (slope $=-0.03(95 \% \mathrm{Cl}:-0.06,0.00, \mathrm{P}=0.04)$, slope difference, $\mathrm{P}=0.11 ;(5)$ decreased obesity among 60 and over aged female from Hunan. Abbreviation: BMI, body mess index; APC, annual percentage change 
A

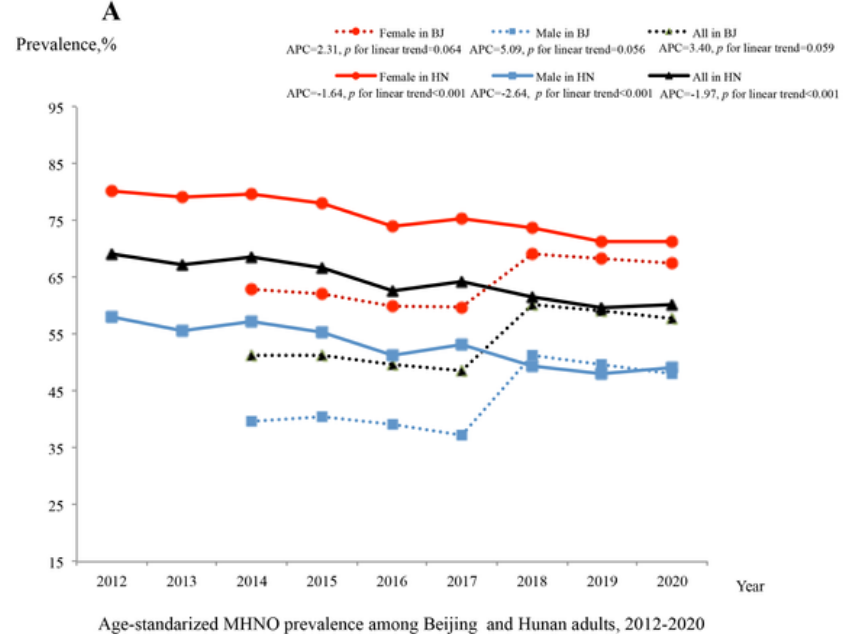

Age-standarized MHNO prevalence among Beijing and Hunan adults, 2012-2020

C

Prevalence, $\%$

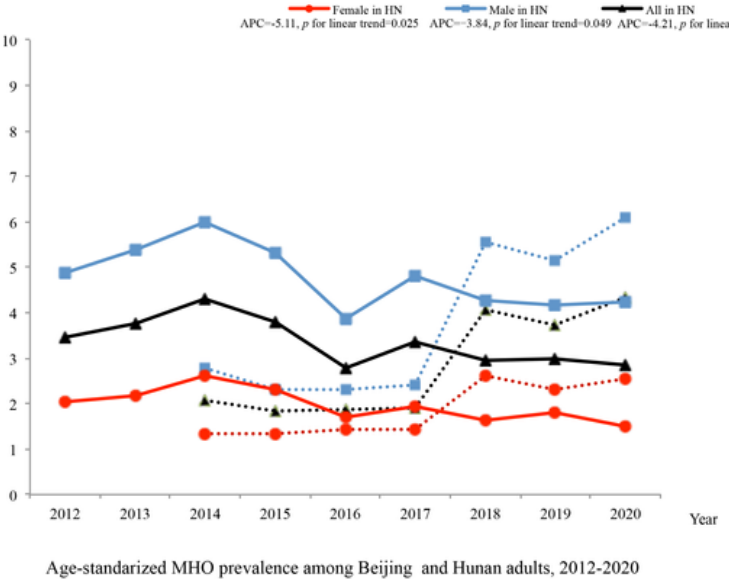

B

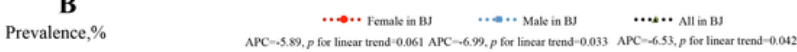

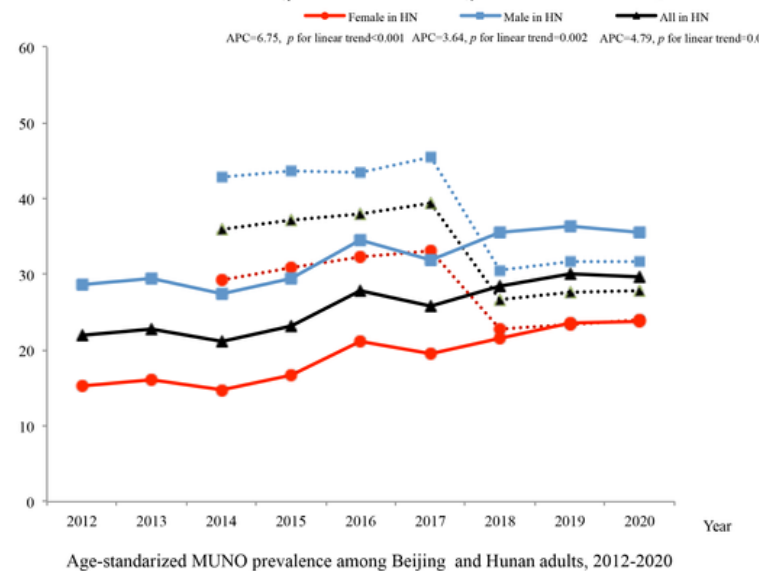

Age-standarized MUNO prevalence among Beijing and Hunan adults, 2012-2020

\section{D}

Prevalence, $\%$

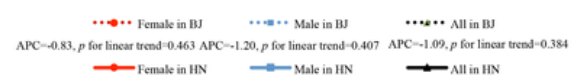

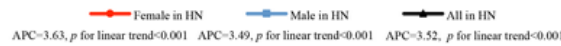

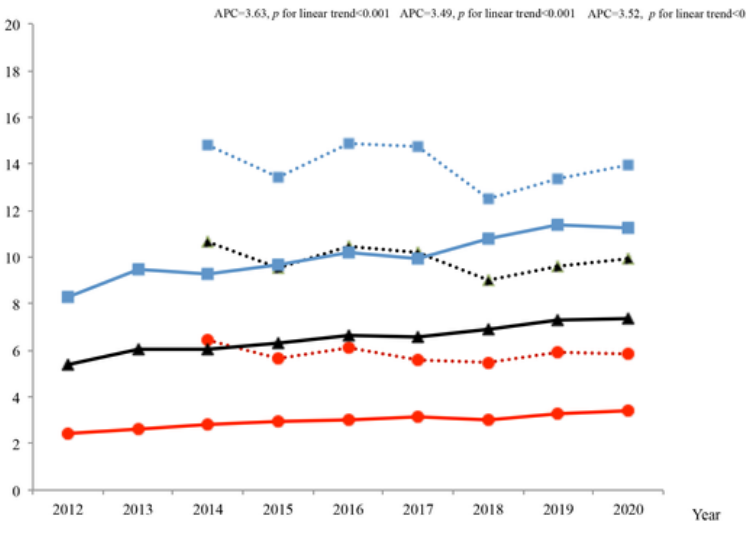

Age-standarized MUO prevalence among Beijing and Hunan adults, 2012-2020

\section{Figure 3}

Trend in prevalence of obesity by metabolic status phenotype in Beijing and Hunan Adults from 2012 to 2020 Estimates by different sex are ageadjusted to the 2010 Chinese Census population using age groups 20-39, 40-59, and 60 or older and estimates for overall population are additional adjusted by sex; Significant linear trends $(P<0.05)$ for the following groups: (1) decreased MHNO among all sex groups from Hunan; $(2)$ decreased MUNO among male and overall groups from Beijing $(P<0.05)$; increased MUNO among all sex groups from Hunan $(P<0.05)$; $(3)$ increased $M H O$ among all sex groups from Beijing and decreased MHO among all sex groups from Hunan; (4) increased MUO among all sex groups from Hunan ( $P<0.05)$. Abbreviation: MHNO, metabolic healthy non-obese; MUNO, metabolic unhealthy non-obese; MHO, metabolic healthy obese; MUO, metabolic unhealthy obese; APC, annual percentage change

\section{Supplementary Files}

This is a list of supplementary files associated with this preprint. Click to download.

- FigS1.pdf

- FigS2.pdf

- FigS3.pdf

- FigS4.pdf

- FigS5.pdf

- FigS6.pdf

- FigS7.pdf

- FigS8.pdf

- STROBEchecklistcrosssectional.docx 
- Supplementalmaterial.docx

- TableS16.docx

Page 12/12 